\title{
IONOSPHERIC DISTURBANCES EXCITED BY THE LITHOSPHERIC GAS SOURCE OF ACOUSTIC GRAVITY WAVES BEFORE EARTQUAKES
}

\author{
Gotynyan O. E. ${ }^{1}$, Ivchenko V. M. ${ }^{1}$, Rapoport Yu. G. ${ }^{1}$, Parrot M. $^{2}$ \\ ${ }^{1}$ Kiev Taras Shevchenko National University, Kiev, Ukraine \\ ${ }^{2}$ Centre National de la Recherche Scientifique, Orleans Cedex 2, France
}

\begin{abstract}
Many satellite and ground observations point at anomalous ionospheric phenomena associated with seismic activity. It was shown that some ionospheric effects before earthquakes can be explained by acoustic gravity waves (AGW) influence on the ionosphere. One of possible sources of these waves can be greenhouse effect gases penetrating from the lithosphere to near ground atmospheric layer. An accurate numerical model of a lithospheric gas source, excitation and propagation of AGW field to the near-source region with altitudes up to the ionospheric $\mathrm{F}$ region is used. The model includes effective boundary conditions on the ground level and takes into account reactive (non-propagating) AGW modes. Numerical convergence is proven. Presence of reactive modes leads to a change of AGW velocity amplitude at altitude $250 \mathrm{~km}$ by an order of value and in 4 times for AGW with periods 15 min. and 1 hour, respectively. Electron concentration disturbances in the ionospheric Fregfion before earthquakes can reach a value of the order of few dozens percents for AGW with period 1 hour, which agrees with the results of observations. Ionospheric response to AGW, namely, relative change of electron concentration at altitude $250 \mathrm{~km}$ reaches a maximum for AGW with period equal to $73 \mathrm{~min}$ for a given (bell-shaped) spatial dstribution of lithospheric gas source with width $100 \mathrm{~km}$. Spatial distribution of electron concentration disturbances are non-symmetrical in the oblique geomagnetic field. "Twin-source" of AGW in the lithosphere can pronounce itself in a splitting of spatial distribution of relative change of electron concentration..
\end{abstract}

\section{Introduction}

An interest to a problem of lithospheric-ionospheric coupling has considerably grown during the last years. It is explained by a many observation data of ionospheric disturbances during preparation of earthquakes such as observations of variations of night sky oxygen emission variations in the spectral lines $557.7 \mathrm{~nm}$ and $630 \mathrm{~nm}[1,2,3]$ at altitudes around 100 and $270 \mathrm{~km}$, respectively, total electron content (TEC) variations [4], the infrared radiation from regions of seismic activity registered from satellites [5], [6], traveling ionospheric disturbances (TID) [7] etc. All of these phenomena can be connected with AGW in one way or another [1-6]. The coupling in the system Lithosphere-Atmosphere-Ionospere-Magnetosphere (LAIM) before strong earthquakes can be realized through electromagnetic [8-10], electrostatic-photochemistry $[11,12]$ and AGW $[6,13]$ mechanisms. The present paper is dedicated only to the last one.

The general objective of our model of the AGW channel of seismo-ionospheric coupling is to consider step by step, "from the bottom to the top" phenomena occurring before earthquakes on the different "floor" of the LAIM system, Fig. 1. This Figure illustrates phenomena, which, as far as is known up to now [6, 13-16], can occur due to AGW excitation in the process of earthquake preparation. On the "first floor", from the lithosphere to the Eregion of the ionosphere, AGW are excited by the heat source of lithospheric green-effect 
gases, the flow of which is modulated by seismogravity Earth oscillations with characteristic periods of order of 1-2 hours [17,6], typical for long-wave branch of AGW, namely internal gravity waves or just gravity waves (GW) [18], Fig. 1. GW with shorter periods (of order of few dozens of minutes) and acoustic waves (AW), a short-period branch of AGW [18] also can be excited by the lithospheric source of AGW [15], Fig. 1. AGW can modify sporadic Elayers $\left(\mathrm{E}_{\mathrm{S}}\right),[14]$, heat atmosphere and cause change of turbulent diffusion coefficient, $D_{\text {turb }}$, and spatial redistribution of atmospheric spices concentrations including O, NO [14] and electron concentration at the altitudes in the range $85 \sim 100 \mathrm{~km}$. As a result, an average increase of the airglow $557.7 \mathrm{~nm}$ intensity [3] can be observed few hours- couple of days before an earthquake [1, 3 ]. Also, due to electron's concentration spatial redistribution, change of effective height of the ionosphere and ionospheric conductivity can occur and, as a result, characteristics (phase and amplitudes) of electromagnetic waves (EMW) propagating in the waveguide Earth-Ionosphere can change before an earthquake [19], Fig. 1. On the "second floor" of the LAIM system, in the F-region, AGW radiated from the lithosphere can cause periodical variations with periods 1-2 hours (1-2 days before earthquake), and/or change of average value of the electron concentration and of ionospheric critical frequency, $f_{0}$ (2-3 days before earthquake) [20]. Also, AGW, including those of the lithospheric origin, reaching near-equatorial ionospheric F-region, can become a seeding perturbation for development of a chain of "triggering" phenomena such as: Rayleigh-Tailor instability (RTI, see Fig. 1) -grows of plasma disturbances (and probably of AGW as such)-development of plasma bubbles-development of the plasma microturbulence with variations of electron concentration $\delta n$, electrostatic field, $\delta E$, and electron $\delta T_{e}$, and ion, $\delta T_{i}$ temperatures [21], Fig. 1. Satellite observations of plasma turbulence and of modification of the equatorial anomaly before earthquake have been reported recently [16]. Probably, vortex development along with floating-up of plasma bubbles [22] could also be observed in the F-region of the ionosphere before earthquakes, Fig. 1. Time and space scales of the structures observed before earthquakes correspond to the typical parameters of the AGW [23, 24]. Typical dimensions of a lithospheric source and of region in the ionosphere where phenomena described above are observed are few dozens-few hundred and few thousand kilometers, respectively (Fig. 1). It is possible to guess that "higher floors" of the system LAIM could be "occupied" by VLF radiation from the regions of plasma turbulence, propagating along geomagnetic field lines [15]. Present paper and [13] describe only few (but important) features of the general picture of seismoionospheric (and, more generally, thermospheric-ionospheric and/or lithospheric-ionospheric) coupling phenomena illustrated by Fig. 1.

An accurate model of "AGW antenna" and of AGW excitation by the lithospheric green-house effect gases before an earthquake have been proposed first in [13]. In the present papers, some details of calculation of the AGW field in the near-source region are presented with emphasize on the effect of reactive (non-propagating) modes, and the linear effect of AGW of the lithospheric origin on the ionospheric F-region with the oblique geomagnetic field is considered. 


\section{The model of the AGW lithospheric gas source}

The set of hydrodynamics equations describing AGW with a heat source, source of mass and a force source has the form [18]

$$
\begin{gathered}
\rho \frac{d \overrightarrow{\mathrm{v}}}{d t}=-\vec{\nabla} p+\rho \vec{g}+\rho \vec{F}, \\
\frac{d \rho}{d t}+\rho \operatorname{div} \overrightarrow{\mathrm{v}}=Q, \\
\frac{d p}{d t}+\overrightarrow{\mathrm{v}} \vec{\nabla} p=c^{2}\left(\frac{\partial \rho}{\partial t}+\overrightarrow{\mathrm{v}} \vec{\nabla} \rho\right)+A \rho,
\end{gathered}
$$

where $\rho, \overrightarrow{\mathrm{v}}, p, \vec{g}$ and $c$ are density, velocity and pressure of neutral atmosphere and gravity acceleration, respectively, $d / d t \equiv \partial / \partial t+\overrightarrow{\mathrm{v}} \vec{\nabla}, \vec{e}_{z}$ and $\vec{F}=F_{z} \vec{e}_{z}$ are unit vertical vector (in $z$ direction) and the force function, respectively, $Q(\vec{r}, t)$ and $\grave{A}(\vec{r}, t)$ are densities of the mass and heat sources respectively which are determined by gases released from the lithosphere. In [6], the system (1) was solved for GW, long-period branch of AGW, without taking into account non-propagating (reactive) modes and with step-like source without specifying boundary conditions. An accurate model for the excitation of AGW with derivation of effective boundary conditions on the Earth surface was built first in [13]. Geometry of the problem is shown in Fig. 1b. Curvature of the Earth surface is not taken into account and a model of homogeneously stratified atmosphere is used. Stationary problem of AGW excitation with some frequency $\omega$ is considered. Physically this corresponds to a stationary modulation of gas flow, for example, by seismogravity oscillations [6. 14, 17]. Detail description of the source model can be found in [13] and is omitted here. Effective boundary conditions at the ground level are derived on the basis of consideration of the three-layered model including two semi-infinite layers of Earth and atmosphere and thin near-ground gas layer containing green-house effect gases, located between two semi-infinite layers [13]. Periodical lateral (zero) boundary conditions are used for the AGW field on the distance far enough from the region of interest, to use discrete instead of continuous Fourier spectrum of AGW. The upper boundary condition is a condition of AGW radiation. Estimations [13] have shown, that the main contribution to a generation of AGW is due to a heat source (in the right-hand side of the third equation of the system (1)). The spatial distribution of a vertical velocity excited by the 1D lithospheric gas source at the ground level $(z=0)$ is taken in the form $[6,13]$

$$
\mathrm{v}_{\mathrm{z}}^{\mathrm{src}}(x) \approx \frac{\Delta z A}{g H} \approx \frac{\Delta z}{g H} \frac{\omega k_{b} \Delta T}{M} \operatorname{ch}^{-2}\left(\frac{x}{l}\right),
$$

where $x$ is the horizontal coordinate, $\Delta T(\sim 3 \mathrm{~K})$ is the amplitude of the temperature anomaly, caused by green-effect gases, $l(\sim 100 \mathrm{~km})$ is the lithospheric source width, $\Delta z(\sim 3 \mathrm{~m})$ is a thickness of near-ground gas layer, containing green-effect gases (such as $\mathrm{CO}_{2}$ ), $T=2 \pi / \omega$ 
is a period of the lithospheric gas source (or of corresponding lithospheric gas flow modulation), $k_{B}$ and $H$ are Boltzman constant and atmosphere scale high, respectively, $M$ is an average mass of neutral atmosphere particles. An importance of so-called reactive (nonpropagating) modes is connected with the following properties of AGW. AGW dispersion law is [18]

$$
\omega^{2}=\frac{c^{2}}{2}\left(k_{x m}^{2}+k_{z m}^{\prime 2}+\frac{1}{4 H^{2}}\right) \pm \sqrt{\frac{c^{4}}{4}\left(k_{x m}^{2}+k_{z m}^{\prime 2}+\frac{1}{4 H^{2}}\right)-(\Gamma-1) g^{2} k_{x m}^{2}}
$$

or

$$
k_{z m}^{\prime}= \pm \sqrt{k_{x m}^{2}\left(\frac{\omega^{2}}{\omega_{b}^{2}}-1\right)+\frac{\omega^{2}}{c^{2}}-\frac{1}{4 H^{2}}}
$$

where $m$ is number of the mode under consideration

$$
k_{z m}=\frac{i}{2 H}+k_{z m}^{\prime}
$$

$k_{\mathrm{xm}}$ and $k_{\mathrm{zm}}$ are horizontal wave number and a real part of vertical wave number, respectively, $\omega_{b}=\frac{g \sqrt{\gamma-1}}{c}$ is the Brunt-Vaisala frequency, the component $\frac{i}{2 H}$ of a vertical wavenumber describes the effect of atmosphere stratification in the gravity field. As follows from (3b), for modes with horizontal wavenumber satisfying the condition

$$
4 H^{2} k_{x m}{ }^{2}<\left(1-\frac{4 H^{2} \omega^{2}}{c^{2}}\right) /\left(\frac{\omega_{b}^{2}}{\omega^{2}}-1\right)
$$

components $k_{z m}^{\prime}$ of vertical wave numbers, $k_{z m}$ are purely imaginary and corresponding modes are "reactive". These modes can not propagate in the atmosphere. Because horizontal Fourier spectrum is discrete, horizontal (and vertical, for fixed frequency) wave number is discrete as well, $k_{x} \equiv k_{x m}=\frac{2 \pi m}{L}, m=\overline{1-N_{x} / 2, N_{x} / 2}$, where $m$ and $N_{x}$ are, respectively, a number of the horizontal mode under consideration and total number of modes taken into account in the discrete Fourier transform. Here $L$ is the horizontal dimension of the region under consideration, bounded by fictitious lateral walls, chosen in such a way, that $L>l, L>L_{0}$, and these walls does not influence on the AGW field in the "region of interest", where AGW field and ionospheric disturbances are calculated, with the horizontal and vertical dimensions $H_{0}$ and $L_{0}$, respectively. In our calculations $H_{0}=250 \mathrm{~km}$ and $L_{0}$ is of the order of few thousands kilometers (see Figs. 2-10 in the Sect. 4). For the source in the form (2), the reactive modes of $\mathrm{AGW}$ with frequencies $\omega<\omega_{b}$ (in other words, GW) have the lowest numbers $m$ and are excited most effectively at the ground level. During propagation into the atmos- 
phere and the ionosphere these modes decay exponentially. At the same time, effectiveness of the excitation of others modes depends on the amplitudes and phases of reactive modes in spite of that the last cannot propagate to large altitudes. Hence, one can expect that reactive modes are important for excitation and propagation into the ionosphere of GW with finite frequencies. Total number of reactive modes depends on GW frequency. The amplitude of the source (2) is also frequency-dependent. The effect of reactive modes on GW propagation and their influence on the ionosphere is illustrated quantitatively in the Sect. 4.

\section{Influence of AGW on the ionosphere}

In accordance with drift approximation [25, 7, 2], inertialess ion (and electron) concentration, $N$, under conditions of neutrality and

$$
\frac{m_{e} v_{e n}}{e H_{0}}<<\frac{m_{i} v_{i n}}{e H_{0}}<<1,\left|e N \vec{u}_{i}\right|<<\frac{m_{e} v_{e n}}{m_{i} v_{i n}}\left|\vec{j}^{\|}\right|
$$

is governed by the equation

$$
\frac{\partial N}{\partial t}=-\nabla\left(N \overrightarrow{\mathrm{u}}_{\mathrm{i}}\right)+S-L
$$

Here

$$
\vec{u}_{i} \approx \vec{u}_{i}^{\|}=\overrightarrow{\mathrm{v}}^{\|}-\frac{D_{a}}{2 k_{B} T_{i}}\left[\vec{\nabla}^{\|}\left(p_{e}+p_{i}\right)-N m_{i} \vec{g}^{\|}\right]
$$

$e, m_{e, i}, v_{e n, i n}$,are electron charge, electron and ion mass and electron-neutral and ion-neutral collision frequencies, respectively, $\vec{u}_{i}$ is ion drift velocity, index “"\|” means projection on the direction of geomagnetic field, $p_{e, i}$ are electron and ion pressure, respectively, $\vec{j}$ is plasma current, $D_{a}, T_{i}$ are ambipolar diffusion coefficient and ion temperature, respectively. $S$ and

$$
L=\alpha N_{N}\left(N-N_{e 0}\right)
$$

are electron production and loss rates, respectively, $N_{e 0}$ is steady-state concentration of charged particles in plasma. For the case of isothermal electrons and ions, $T_{e}=T_{i}$, ambipolar diffusion coefficient is $D_{a}=2 k_{B} T_{i} /\left(m_{i} v_{i n}+m_{e} v_{e n}\right) \approx 2 k_{B} T_{i} / m_{i} v_{i n}$,

$$
\begin{gathered}
v_{\text {in,en }}=q_{\text {in }, e n} N_{N}, \\
N_{N}=\rho / M
\end{gathered}
$$

is a concentration of neutral particles, coefficients $\alpha$ and $q_{\text {in,en }}$ are described in [26] and [27], respectively.

Linearization of eqs. (4b)-(7a) gives (for night conditions, when $S=0$, or neglecting disturbances caused by the presence of $S$ in eq. (4b) ) 


$$
\begin{aligned}
& \tilde{N}_{e m} \equiv \tilde{N}_{e}\left(k_{m}\right)=\frac{G}{D}, \\
& G=-\left[D_{B} N_{e 0}+\frac{\partial N_{e 0}}{\partial z} \cos \theta\right] \mathrm{v}_{B}+\hat{L}_{1} \tilde{N}_{N}+\alpha N_{e 0} \tilde{N}_{N}, \\
& D=D_{t}-\frac{D_{a}}{2} D_{B}{ }^{2}+\frac{1}{2 H}\left(\frac{\partial D_{a}}{\partial z}+D_{a} D_{z}\right)+\alpha N_{N 0}
\end{aligned}
$$

Here $\tilde{N}_{e}=N-N_{e 0}, \widetilde{N}_{N}=N_{N}-N_{N 0}, N_{N 0}$ is steady-state concentration of neutral particles, $\mathrm{v}_{B}$ is $\mathrm{AGW}$ velocity projection on the geomagnetic field direction, $D_{t}=i \omega, D_{z}=-i k_{z m} D_{B}=\vec{e}_{b} \cdot \vec{\nabla}=-i\left(e_{b x} k_{x m}+e_{b z} k_{z m}\right), \vec{e}_{b}$ is unit vector along geomagnetic field, $\theta$ is angle between geomagnetic field and vertical direction, $\hat{L}_{1} \tilde{N}_{N}$ describes an influence of AGW on the frequency of collisions between charged particles and neutral and on the recombination intensity,

$$
\hat{L}_{1} \tilde{N}_{N} \equiv\left[\frac{D_{a}}{2} \frac{\partial N_{e 0}}{\partial z} \frac{1}{N_{e o}} e_{z B} D_{B}+\frac{1}{2} \frac{\partial}{\partial z}\left(D_{a} \frac{1}{N_{N 0}} \frac{\partial N_{e 0}}{\partial z}\right)\right] \tilde{N}_{N}+\frac{1}{2 H} \frac{D_{a}}{N_{N 0}} \frac{\partial}{\partial z}\left(N_{e 0} \tilde{N}_{N}\right) .
$$

Note that it is straightforward, using linearized form of the system (1) and eq. (7b), to express a value $\tilde{N}_{N}$ through a neutral particle's velocity projection, $\mathrm{v}_{B}$. After collecting components of Fourier transform, one can get using (8):

$$
\frac{N_{e}^{\prime}}{N_{e 0}}\left(x_{n}, z\right)=e^{z /(2 H)} \sum_{m=-N x / 2}^{N x / 2}\left[\widetilde{N}_{e m} / N_{e 0}\right] \cdot e^{-i k_{x m} x_{n}-i k_{z m} z},
$$

where $x_{n}=L \frac{n}{N_{x}}, n=\overline{1-N_{x} / 2, N_{x} / 2}$ is integer number.

\section{Results of numerical calculations}

We emphasize first some peculiarities of AGW field taking into account definite shape and spectrum characteristics of the lithospheric gas source. In the present paper, we restrict ourself only with searching one mechanism of AGW influence on the ionospheric electron concentration, namely carrying out of charged particles by neutral AGW "wind". To do this, we exclude the effects of AGW influence on recombination and collision frequency and put in the present calculations $\hat{L}_{1}=0, \alpha=0$. Plasma parameters of the ionospheric $\mathrm{F}$ region are taken from $[2,7,25]$. Figs. 2, 3 illustrate an influence of the reactive modes on the penetration of the wave field through the atmosphere. Fig. 2 shows, that, in accordance with the condition (3c), the number of reactive modes increases with decreasing AGW frequency (below the Brunt-Vaisala frequency). For frequencies larger than Brunt-Vaisala frequency, there are no reactive modes. Fig. 2 shows a result of multiplication of amplitudes, $V_{m}$ of the modes of lithospheric source with different horizontal wavenumbers, $k_{x m}$, by exponential factor, 
$\exp \left(-i k_{z m} z\right)$, where $k_{z m}$ is determined by $(3 \mathrm{~b}, \mathrm{c})$. For reactive modes (see $\left.(3 \mathrm{~b}-\mathrm{d})\right), k_{z m}$ are imaginary, therefore reactive modes decay exponentially with altitude, relatively to nonreactive modes (for which $k_{z m}$ are purely real). At the same time, reactive modes correspond to the maximum of spectral distribution of the symmetrical source under consideration. By this reason, reactive modes are of a great importance for the characterization of an influence of the lithospheric source of $\mathrm{AGW}$ on the ionosphere. In calculations [6], the term $\frac{\omega^{2}}{c^{2}}-\frac{1}{4 H^{2}}$ in expression ( $3 b$ ) was neglected, what is valid only for GW modes with very small frequencies, $\omega$ and large enough horizontal wavenumbers, $k_{x m}$. As seen from (3b, c), in this case reactive modes do not exist at all, in other words, values $k_{z m}$ 'are real. Inscriptions "taking into account reactive mode" and "with reactive modes" in Fig. 3 mean that we do not neglect the term $\frac{\omega^{2}}{c^{2}}-\frac{1}{4 H^{2}}$ in $(3 b)$ and therefore, in our model some of modes are reactive (solid curve in Fig. 3). For comparison, we made also calculations in the approximation [6], "without reactive modes" (dashed curve if Fig. 3). It is seen, that amplitudes of vertical velocity, $\left|\overrightarrow{\mathrm{v}}_{z}\right|$, at altitude $250 \mathrm{~km}$, determined using accurate dispersion low $(3 \mathrm{a}, \mathrm{b})$ and in approximation [6] ("without taking reactive modes into account") differ for AGW with period $15 \mathrm{~min}$ by more than one order of value. For AGW with period $60 \mathrm{~min}$, this difference reaches a value about 4 times. Fig. 4 shows qualitative difference in spatial distributions of vertical velocity normalized to its maximum value for $\mathrm{AGW}$ with frequencies equal to and lower than Brunt-Vaisala frequency (corresponding periods are $5.8 \mathrm{~min}$ and 15 and $60 \mathrm{~min}$, respectively). These distributions have one and two spatial peaks in the first and second cases respectively. Figs. 5,6 illustrate spatial distributions of absolute values of vertical and horizontal velocity amplitudes for periods 15 and $60 \mathrm{~min}$. It is seen that for AGW with period $15 \mathrm{~min}$, maximum values of amplitudes of horizontal and vertical velocities are comparable, and for AGW with period $60 \mathrm{~min}$, maximum value of horizontal velocity exceeds sufficiently (in few times) maximum value of vertical velocity at altitude $250 \mathrm{~km}$. Respectively, maximum values of relative change of electron concentration caused by AGW with period 60 min exceeds sufficiently corresponding value for $\mathrm{AGW}$ with period $15 \mathrm{~min}$ (figs. $7 \mathrm{~b}$ and $7 \mathrm{a}$, respectively).

Drift of plasma along geomagnetic field in the F- region of the ionosphere causes remarkable non-symmetry in the spatial distribution of the change of electron concentration in the AGW field, which is more prominent for the AGW with smaller period (Fig. 7). The typical value of relative change of electron concentration (of order of few dozens of percents) and of spatial scales of a region in the ionosphere where excitation can reach (of order of thousand kilometers) coincides qualitatively with the results of observations in the F region of the ionosphere before earthquakes (see [20] and [23], respectively). In Fig. 8, dependence of maximum relative value of change of electron concentration $N_{e}{ }^{\prime \max } / N_{e 0}$ on a period of $\mathrm{AGW}$ is shown (at an altitude $z=250 \mathrm{~km}$ ). For each period, $N_{e}{ }^{\prime \max } / N_{e 0}$ is the value of maximum of 
corresponding spatial distribution. Ripples in the dependence shown in Fig. 8 correspond to the finite numerical accuracy of determination of maximum using spatial Fourier transform with finite number of spatial modes. A presence of maximum in frequency dependence of $N_{e}{ }^{\prime \max } / N_{e 0}$ is explained as follows. It is seen from (8) that if $\omega \rightarrow 0(T \rightarrow \infty)$, then $k_{z} \sim 1 / \omega$. Taking into account that under this condition media is practically incompressible, one can get for any definite mode (with number $\mathrm{m}$ ):

$$
\mathrm{v}_{z m} k_{z m}+\mathrm{v}_{x m} k_{x m}=0 .
$$

Taking into account (2), (10), it is easy to see, that under these conditions, $\mathrm{v}_{x} \rightarrow$ const, $\mathrm{v}_{z} \rightarrow 0$, where $\mathrm{v}_{x, z}$ are components of the $\mathrm{GW}$ velocity. This tendency is also proven by numerical calculations. In accordance with (8), (2), (10), one can get that $N_{e}{ }^{\prime \max } / N_{e 0} \sim \omega$, when $\omega \rightarrow 0$. At the same time, it is easy to see from (2), (3) that $N_{e}{ }^{\prime \max } / N_{e 0} \rightarrow 0$, if $\omega \rightarrow \infty(T \rightarrow 0)$. Therefore, the existence of maximum in dependence $\left[N_{e}{ }^{\prime \max } / N_{e 0}\right](T)$ is obvious. We should point out that this dependence is a characteristic of both lithospheric source as a whole and of (ideal, in our case) stratified atmosphere (more accurately, of its layer between $z=0$ and $z=250 \mathrm{~km}$ ) as a filter of AGW. Also it is interesting that a maximum of the dependence $\left[N_{e}{ }^{\prime m a x} / N_{e 0}\right](T)$ corresponds to a period of AGW 73 min., rather close to typical periods of variations of electron concentration in the F-region of ionosphere (which are of order of 1 hour). A vertical line in Fig. 8 at period about 100 min., separates region of AGW periods for which the present approximation of the "plane geometry" is valid, from a region of larger AGW periods where an influence of the Earth curvature should be taken into account; at least we expect that for AGW with such periods "effect of curvature", not taken into account in the present work, can be noticeable).

All Figs. 2-8 are built for a single lithospheric source with a shape described by eq. (2). As shown in [5], in some cases (at least) two regions with temperature anomalies were observed in the lithosphere (on the ground level) before earthquakes. These sources could be "two-dimensional" earthquake preparation regions or two "one-dimensional" faults in the Earth crust (like "twin" Kopetdag fault (see [5], Fig. 5). Another example of possible "multiple" source of AGW in the lower atmosphere could be few propagating vortexes. An examples of "twin" thermal source (or two neighboring sources) in the lithosphere (at $z=0$ ) and corresponding ionospheric responce are shown in Fig. 9, 10, respectively. Solid line in Fig. 10 corresponds to AGW with period 73 min. of maximal ionospheric response (shown in Fig. 8). For such a period, ionospheric responce from "twin source" (Fig. 9) reveals in appearance of splitting in spatial distribution of relative change of electron concentration (solid curve in Fig. 10). For smaller periods (dashed curve in Fig. 10), such a splitting does not appear (spatial shape of lithospheric source with period $30 \mathrm{~min}$, is the same as for source with period $73 \mathrm{~min}$. , shown in Fig. 9). 


\section{Discussion and conclusions}

Spatial spectrum and frequency of definite source of AGW in the lithosphere are important for the subsequent development of the disturbances in the ionosphere, in accordance with scheme in Fig. 1. AGW can be a seeding factor for development of Rayleigh-Taylor [28, 26] or quasistatic gradient-drift instability [29] with subsequent development of plasma bubbles. Another possibility of "trigger effects" is a development of Perkins instability [30, 31]. It is important that plasma "eigenwaves" of Rayleigh-Taylor [28] and Perkins [30, 31] instability can be "in resonance" with AGW in particular in the presence of neutral wind. "Autooscillations" which can develop on the nonlinear stage of the quasi-static gradient-drift instability [29] have a period 1 hour, closed to typical period of AGW. Time evolution of different types of instabilities mentioned above, are described by an equation of the form

$$
d A_{k} / d t+\left(\gamma_{k}^{\prime}+i \gamma_{k}^{\prime \prime}\right) A_{k}=\sum_{n} P_{n} e^{i \omega t}+[\text { Nonlinearity }]
$$

where $A_{k}$ is an amplitude of unstable plasma spectral mode with wavenumber $k, \gamma_{k}{ }^{\prime}, \gamma_{k}{ }^{\prime \prime}$ are linear dumping coefficient and increment of instability, set by $n$ includes spatial spectrum of an "external force", which is AGW of the lithospheric origin in our case. A presence of such a force is important because values of increments of Rayleigh-Tailor and Perkins instabilities at ionospheric F-region are, typically, relatively small $[28,30,31]$. Spectrum $\left(P_{n}\right)$ of such an "external force" in the right-hand part of eq. (11) can be determined using the method proposed in [13] and in the present paper.

We would like to emphasize three aspects of the model of AGW excitation by the lithospheric (gas) source relative to the AGW spectrum at the ionospheric altitudes, important for understanding the mechanisms of "AGW channel" of seismoionospheric coupling. (1) Because, in accordance with model in Fig. 1, a lot of interesting effects including instabilities develop in the $\mathrm{E}$ and lower $\mathrm{F}$ regions of the ionosphere, we should have a model allowing calculations of AGW field in the near-region of "lithospheric antenna". (2) The problem of identification of the time and spatial characteristics of the "lithospheric source" of ionospheric disturbances using both ground-base and satellite observations is actual. Also, possible "resonances" in eq. (11) for plasma instability developing due to "seeding AGW" are very sensitive to the spectral composition of the particular AGW source. Finally, it was shown, for example, in [31], that only modes with wavector orthogonal to the direction of geomagnetic field excite effectively an electric field in the ionosphere. Therefore, we need a model taking into account peculiarities of a shape and, respectively, a spatial spectrum of lithospheric AGW source. (3) In accordance with [28, 29, 31], AGW with wavelength in the range at least from few dozens to few hundred kilometers can be important "seeding factor" for development of Perkins and/or Rayleigh-Tailor instabilities. It was shown above that reactive modes are of a great importance for propagation into the ionosphere of gravity waves, in particular with relatively shorter periods (of order of few dozens minutes) and wavelengths of order of few dozens kms and even up to $\mathrm{AGW}$ (or GW) with period $60 \mathrm{~min}$. In the papers $[15,16]$, a possible important 
role of the AGW with periods of order of few dozens minutes in the dynamics of equatorial anomalies is emphasized. Therefore, we should have a model taking into account reactive modes of gravity waves.

We believe, that an accurate model of the lithospheric gas source satisfying all these requirements and suitable for modeling ionospheric response to the AGW excitations in the lithosphere is developed first in [13] and in the present work. Using this model, we are developing farther modeling energy propagation in accordance with Fig.1, step by step, from the lithosphere, to the subsequent "floors" of the system LAIM. This will include in particular investigation of possible physical effects and comparison of theory with results of ground and satellite observations, including DEMETER, INTERBALL and VARIANT.

Results of [13] and of present paper describe only some (but important) features of the general picture of seismoionospheric (and, more generally, thermospheric-ionospheric and/or lithospheric-ionospheric) coupling phenomena illustrated by Fig. 1. Development of the present model and consideration, "step by step", "from the bottom to the top" of "multi-floor" LAIM system including modeling phenomena shown in Fig. 1, and others thermosphericionospheric phenomena is a subject of future papers.

On the basis of numerical modeling we would like to make the following conclusions.

Numerical model of gas source of AGW is developed, different types of the "excitation force" of AGE are compared and numerical convergence (of order of 1.5\% for 500 spatial modes) is proven

Reactive modes are taken into account. Their presence lead to a change of velocity amplitude at altitude $250 \mathrm{~km}$ by an order of value and in few times for AGW with periods of 15 min and 1 hour respectively.

Values of amplitudes of AGW velocity are of order of few dozens of $\mathrm{km} / \mathrm{s}$ and relative change of electron concentration reaches few dozens of percents at altitudes $250 \mathrm{~km}$ for AGW with periods of order of 1 hour and space scales of order of thousand kms. These characteristics of ionospheric response to AGW excitation coincide qualitatively with the results of observations $[20,23,24]$ for ionospheric F-layer response to the processes of strong earthquake's $(\mathrm{M}>4.5-5)$ preparation.

In the inclined geomagnetic field, spatial distribution of F-layer ionospheric response to the AGW of the lithospheric origin has sufficient asymmetry.

1. Gladishev V. A., Fishkova L. M. Optical research of seismoactivity effects of the ionosphere. In: Electromagnetic phenomena related to earthquake prediction / Ed. M. Hayakawa, Y. Fujinawa,. TERRAPUB Tokyo, pp. 375-380, 1994.

2. Porter H. S., Silverman S. M., Tuan T. F. On the Behavior of Airglow Under the Influence of Gravity Waves // J. Geophys. Res., vol. 79, N 25, pp. 3831-3833, 1974.

3. Toroselidze T. I. Analysis of aeronomy problems using radiation of higher atmosphere (in Russian). "Mecniereba" Publ., Tbilisy, Georgia, 1991.

4. Parrot M. Statistical studies with satellite observations of seismogenic effects, In: Atmospheric and Ionospheric Electromagnetic Phenomena Associated with Earthquakes / Ed. M. Hayakawa, TERRAPUB Tokyo, pp. 685-695, 1999 
5. Gornyj V. I., Sal'man A. G., Tronin A. A., Shylin B. V. Leaving infrared radiation - of seismic activity (in Russian) // DAN USSR. 1988, pp. 301. N. 67-69.

6. Gohberg M. B., Nekrasov A. K., Shalimov S. L. To the influence of nonstable release of green-effect gases in seismically active regions on the ionosphere // Physics of the Earth ("Fizika Zemli", in Russian), N 8, pp.52-55, 1996.

7. Hooke W. H. Ionospheric irregularities produced by internal atmospheric gravity waves // J. Atmosph. Terr. Phys., vol. 30, pp. 795-823, 1968.

8. Molchanov O. A., Hayakawa M., Rafalsky V. A. Penetration characteristics of electromagnetic emission from an underground seismic source into the atmosphere, the ionosphere, and magnetosphere // J. Geophys. Res., 1995, vol. 100A, p. 1691.

9. Grimalsky V. V., Kremenetsky I. A., Rapoport Yu. G. Excitation of electromagnetic waves in the lithosphere and their penetration into ionosphere and magnetosphere // J. Atmospheric Electricity, 1999, vol. 19, N 2, pp. 101-117.

10. Grimalsky V. V., Kremenetsky I., Cheremnykh O. K., Rapoport Yu. G. Spatial and frequency filtration properties of ULF EM radiation of lithospheric origin in the lithosphere-ionosphere-magnetosphere system, in: Seismo Electromagnetics: Lithosphere-Atmosphere-Ionosphere Coupling / Eds. M. Hayakawa and O. A. Molchanov, 2002, pp. 363-370, TERRUPUB, Tokyo.

11. Martinenko S. I., Fuks I. M., Shubova R. S. Ionospheric electric-field influence on the parameters of VLF signals connected with nuclear accidents and earethquakes // J. Atmospheric Electrricity, 1996, vol. 16, N 3, pp. 259-269.

12. Grimalsky V. V., Hayakawa M., Ivchenko V. N., et al. Penetration of an electrostatic field from the lithosphere into the ionosphere and its effect on the D-region before earthquakes // JASTP. 2002. In press.

13. Gotynyan O. E., Ivchenko V. M., Rapoport Yu. G., Model of the internal gravity waves excited by lithospheric greenhouse effect gases // Space Science and Technology (“Kosmichna nauka i tehnologiya”)., Vol. 7, N2., pp. 26-33, 2001.

14. Gohberg, M. B., Shalimov S. L. Lithospheric-ionospheric coupling and its modeling Russian // J. Earth Sciences, vol. 2, N 2, 2000.

15. Molchanov O. A., Hayakawa M., Afonin V. V., et al. Possible influence of siesmicity by gravity waves on the ionospherivc equatorial anomaly from data of IK-24 satellite 1. Search for idea of seismo-ionosphere coupling. In: Seismo Electromagnetics: Lithosphere-Atmosphere-Ionosphere Coupling / Eds M. Hayakawa and O. A. Molchanov. pp. 287-296, TERRUPUB, Tokyo, 2002.

16. Molchanov O. A., Hayakawa M., Afonin V. V., et al. Possible influence of siesmicity by gravity waves on the ionospherive equatorial anomaly from data of IK-24 satellite. 2. Equatorial anomaly and small-scale ionospheric turbulence. In: Seismo Electromagnetics: Lithosphere-Atmosphere-Ionosphere Coupling // Eds M. Hayakawa and O. A. Molchanov, pp. 287-296, TERRUPUB, Tokyo, 2002.

17. Linkov E. M., Petrova L. N., Osipov K. Seismogravity oscillation of Earth and connected with them ionospheric disturbances // DAN USSR, vol. 5, N. 1095-1098, 1990. (in Russian).

18. Hines C.O. Internal atmospheric gravity waves at ionospheric heights // Can. J. Phys., 38. 1441-1481, 1960.

19. Molchanov O. A., Hayakawa M. Subionospheric VLF signal perturbations possibly related to earthquakes // J. Geophys. Res., 1998, 103, A8, pp. 17489-17504.

20. Popov K. V., Liperovskiy V. A., Alimov O. A. Modification of spectra of night i0onospheric F2 layer density variations during the periods of earthquake preparation // Physics of the Earth, 1996, N 1, pp. $93-96$ (in Russian).

21. Ossakov S. Spread-F theories- a review // J. Atmospheric and Terrestr. Phys., 1981, vol. 43, N 5/6, pp. 437452.

22. McDaniel R. D., Hysell D. L. Models and DE observations of internal-regime irregularities in equatorial spread F // J. Geophys. Res., 1997, vol. 102, N A10, pp. 22,233-22,246.

23. Liperovsky V. A., Meister C. V., Popov K. V., et al. On the time scales of some seismo-ionospheric effects // Seismo Electromagnetics: Lithosphere-Atmosphere-Ionosphere Coupling / Eds M. Hayakawa and O. A. Molchanov, pp. 325-327, TERRUPUB, Tokyo, 2002.

24. Meister C. V., Liperovskaya E. V., Molchanov O. A., et al. To the question of spatial scales of seismoionospheric effects. In: Seismo Electromagnetics: Lithosphere-Atmosphere-Ionosphere Coupling / Eds M. Hayakawa and O. A. Molchanov, pp. 329-3331, TERRUPUB, Tokyo, 2002.

25. Kendall, Pickering, Magnetoplazma diffusion at F2-region altitudes // Planet Space Sci, 1967, vol. 15, pp. 825-833.

26. Genkin L. G., Eruhimov L. M., Myasnikov E. N., Shvarts M. M. To the question of generation and buoyancy of isothermal ionospheric and chromospheric "bubbles" // Izvestiya Vuzov Radiofizika (in Russian), vol. 30, pp. 567-577, 1987.

27. Gershman B. N. Dynamics of ionospheric plasma, Moskow, Nauka, 1974. 
28. Huang C. S., Kelley C., Nonlinear evolution of equatorial spread of F2. Gravity wave seeding of RayleighTaylor instability // J. Geophys. Res., A101, pp.293-302, 1996.

29. Sazonov S. V. Nonlinear oscillating regime of electromagnetic disturbances in equatorial region F // Geomagnetism and Aeronomy, 1990, vol. 30, pp. 440-445.

30. Miller C. A. Electrodynamics of midlatitude spread F 2. A new theory of gravity wave electric fields // J. Geophys. Res., 1997, Vol. 102, pp. 11,533-11,538.

31. Huang C.-S., Miller C. A., Kelley M. C. Basic properties and gravity wave initiation of the midlatitude F region instability // Radio Sci., vo;. 29, pp. 395-405, 1994.

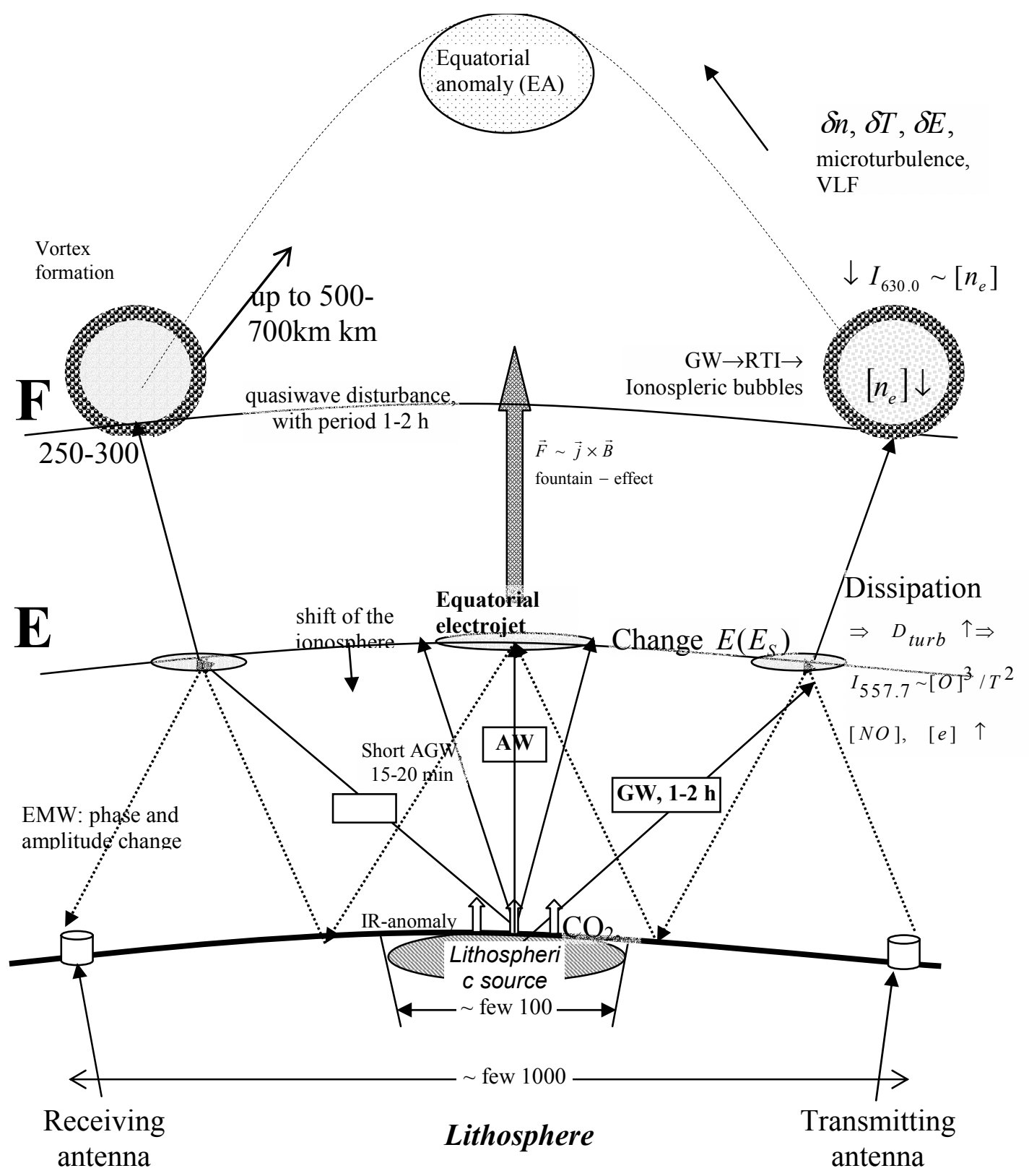

Fig. 1a. Model of AGW channel of seismo-ionospheric coupling 


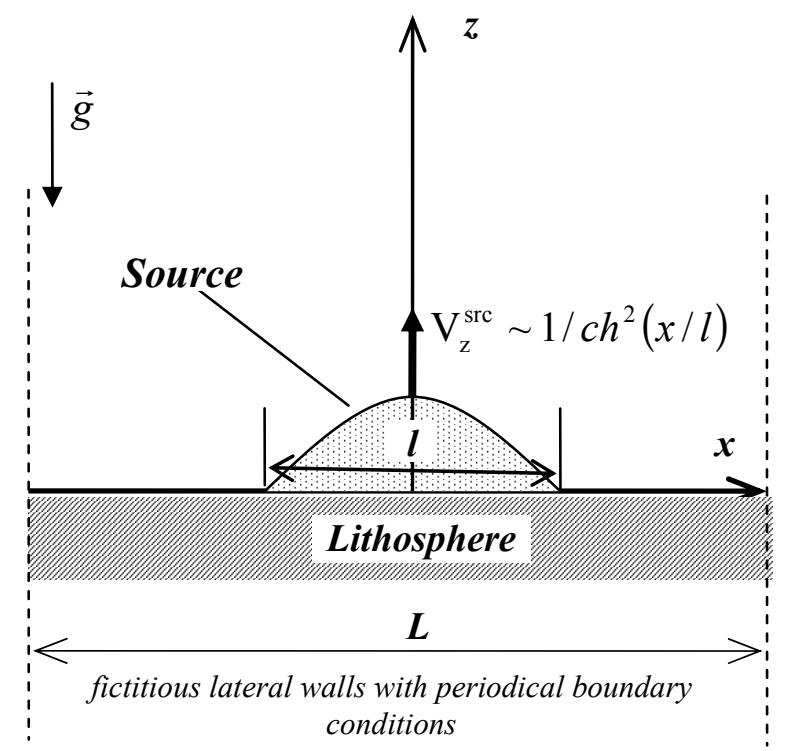

Fig. 1b. Geometry of the problem

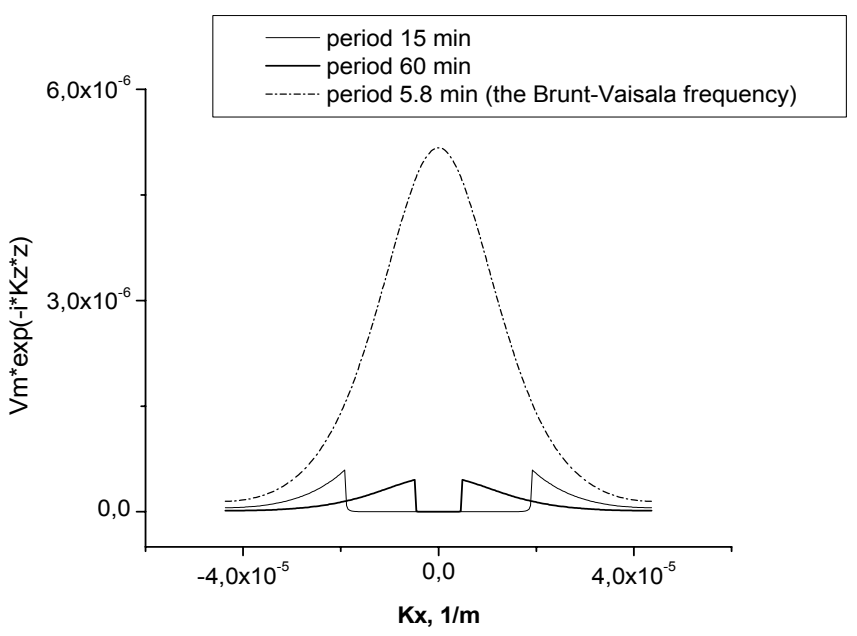

Fig. 2. Dependence of amplitudes of harmonics on horizontal component of wavenumber for different periods of AGW at the altitude $250 \mathrm{~km}$. The gap in the center corresponds to the reactive modes 


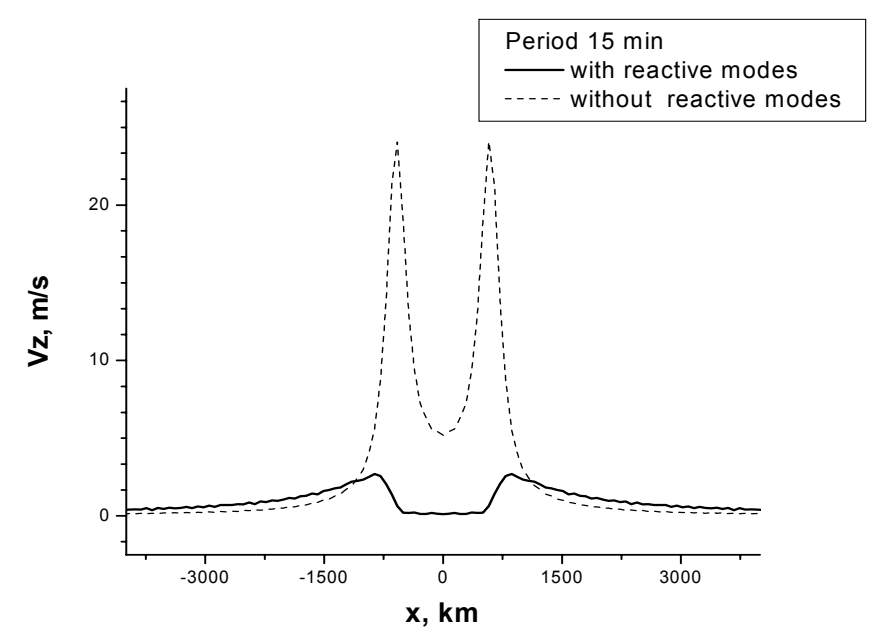

Fig. 3 Influence of reactive modes on the vertical velocity of AGW with period $15 \mathrm{~min}$ at the altitude $250 \mathrm{~km}$

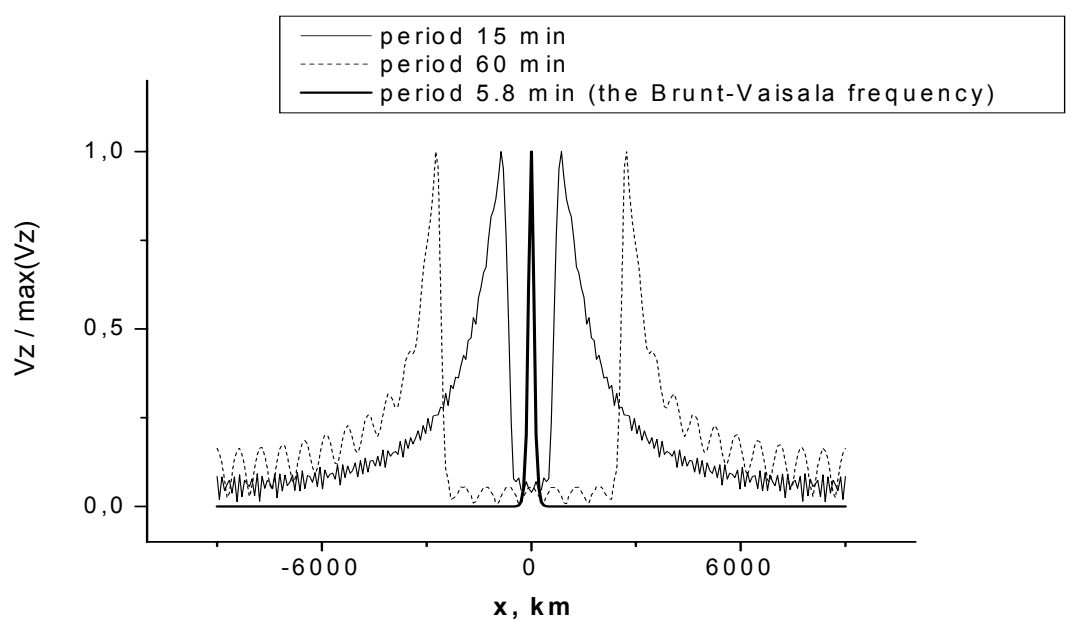

Fig. 4. Vertical velocity of AGW, normalized on the maximum value, for different periods at the altitude $250 \mathrm{~km}$ 


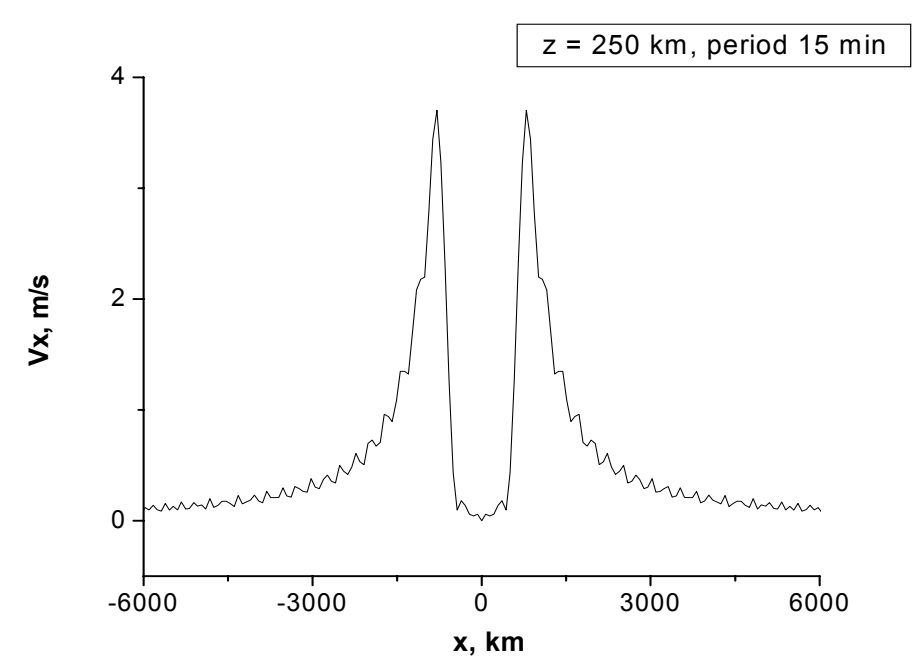

Fig. 5. Field of AGW at the altitude $250 \mathrm{~km}$ for wave period equal to $15 \mathrm{~min}$

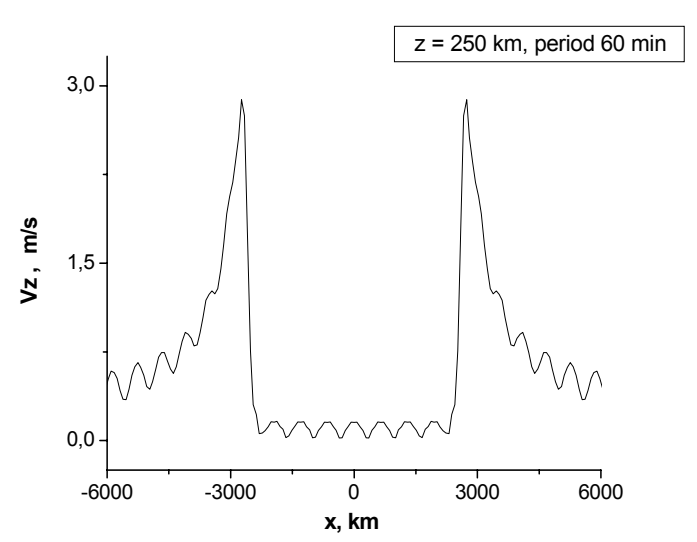

a)

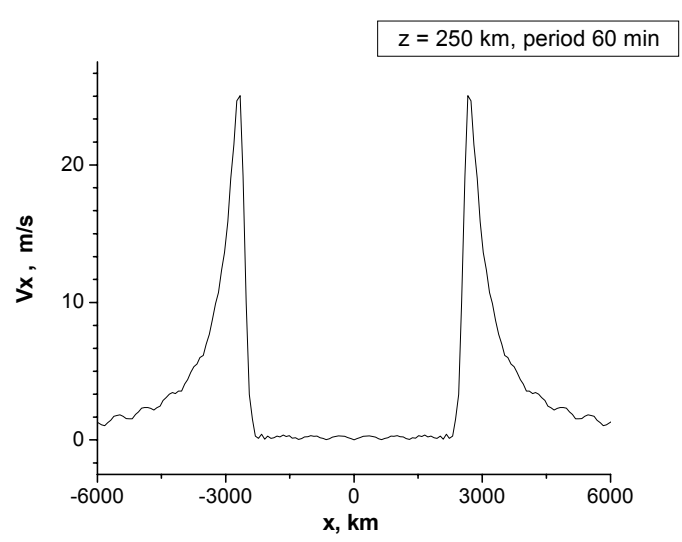

b)

Fig. 6. Velocity of AGW at the altitude $250 \mathrm{~km}$ for wave period equal to $60 \mathrm{~min}$, (a) - vertical velocity, (b) - horizontal velocity 


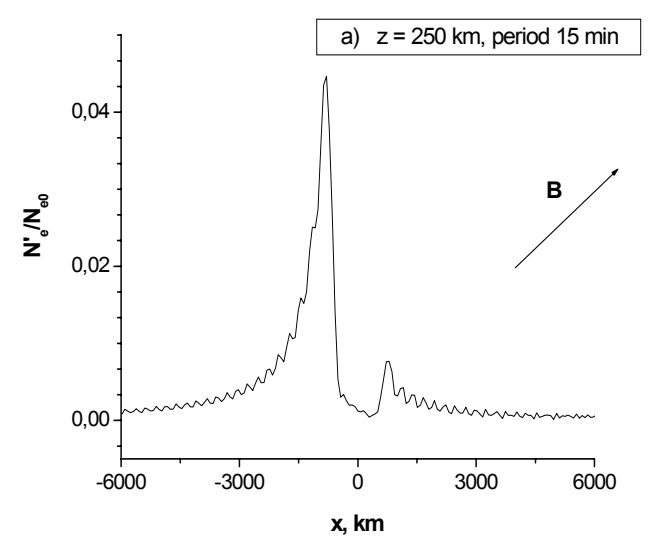

a)

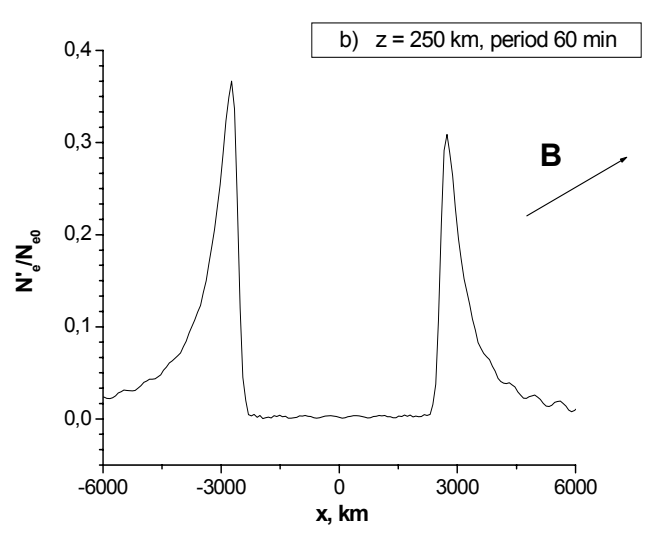

b)

Fig. 7. Space distribution of the relative chance of electron concentration at the altitude $250 \mathrm{~km}$, (a) - period of AGW is $15 \mathrm{~min}$, (b) - period of AGW is $60 \mathrm{~min}$. Angle between magnetic field and vertical direction is equal to $45^{\circ}$

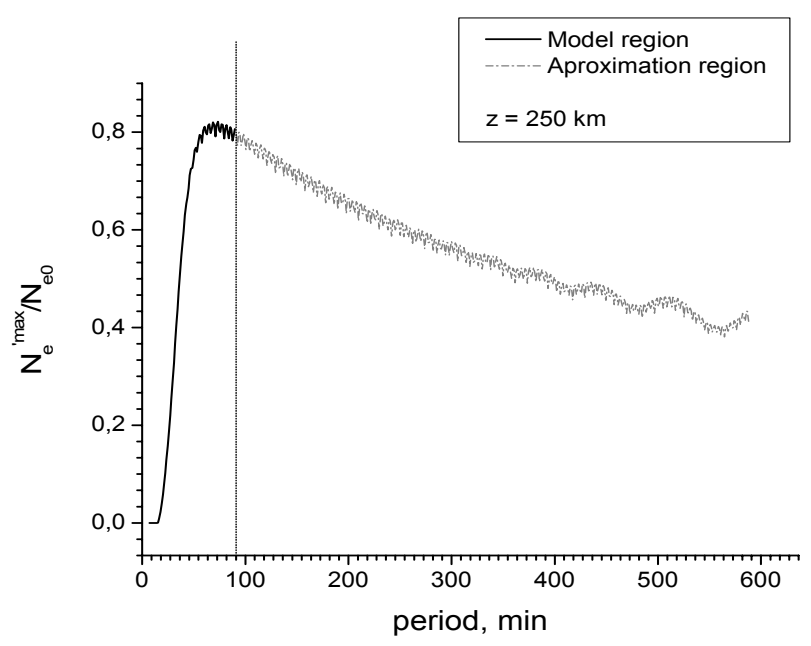

Fig. 8. Dependence of maximum value of space distribution of relative change of electron concentration on AGW period 


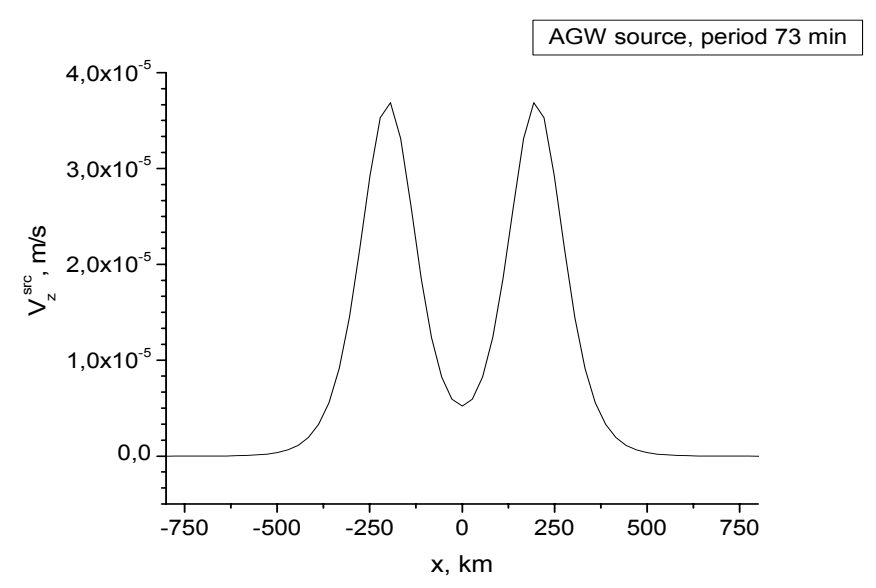

Fig. 9. Spatial distributions of double lithospheric AGW sources

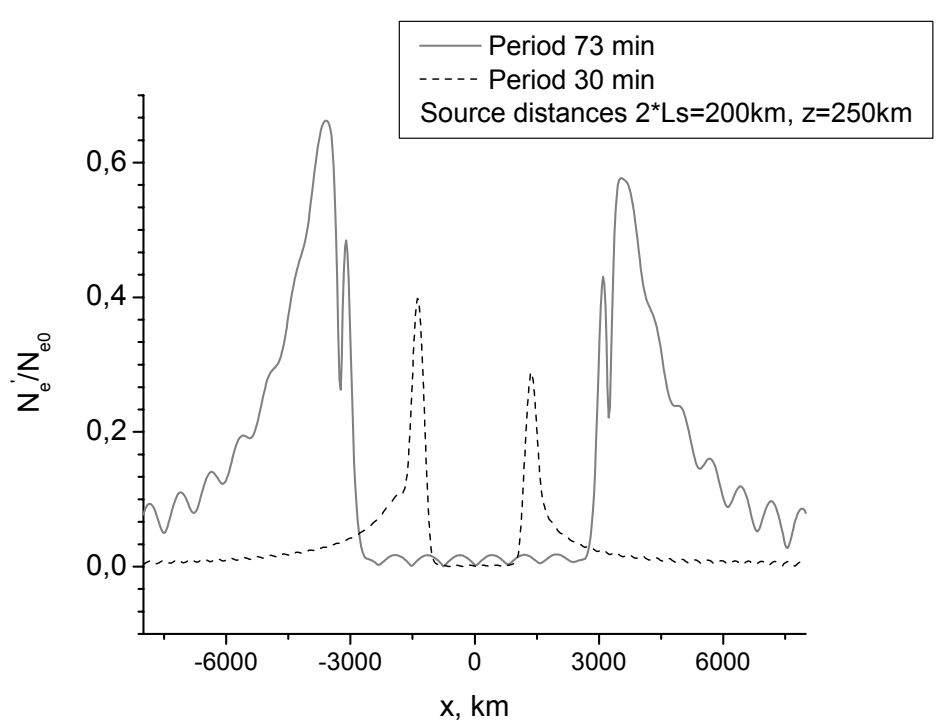

Fig. 10. Space distribution of the relative chance of electron concentration at the altitude $250 \mathrm{~km}$ for periods $73 \mathrm{~min}$ and $30 \mathrm{~min}$ for double source with distance between two sources equal to 2 width of each source $(200 \mathrm{~km})$ 